\title{
A Rare Peritoneal Adenomatoid Tumor
}

\author{
Stefania Erra, Nano Ennio \\ Department of Surgical Pathology, Santo Spirito Hospital, Casale Monferrato, Italy.
}

\begin{abstract}
Corresponding Author:
Dr Stefania Erra

Email: stefania.erra@virgilio.it

This is an Open Access article distributed under the terms of the Creative Commons Attribution License (creativecommons.org/ licenses/by/3.0).
\end{abstract}

$\begin{array}{lll}\text { Received } & : & \text { July 23, 2020 } \\ \text { Accepted } & : & \text { August 13, 2021 } \\ \text { Published } & : & \text { September 30, } 2021\end{array}$

\begin{abstract}
Background: Adenomatoid tumors (AT) are benign tumor arising from mesothelial lining of organs. Genital tract is the most common site of origin for these tumors. These types of tumors are slow-growing, usually asymptomatic (based on the site of growth) and in consequence they can be spotted many years after their initial growth. Case Report: A 59-year-old man presented with a non-painful nodule arising from the anterior omental cavity. The nodule was excised and morphologically evaluated as adenomatoid tumor of the peritoneal cavity. IHC followed to sustain the diagnosis. Conclusion: Microscopic morphology and tumoral immune-phenotype help to distinguish ATs from welldifferentiated adenocarcinomas.
\end{abstract}

Keywords: Adenomatoid tumor, Mesothelium, Immmunohistochemistry, Peritoneum.

\section{Introduction}

Adenomatoid tumors (AT) are benign tumors affecting mainly genital tracts in both male and females, in which they have the same incidence [1]. Although AT mainly affects genital organs, there have been reported cases of AT (like ours) arising from extra-genital sites like: meso-colon, liver, pancreas, lymph nodes, heart, and adrenal glands [2].

AT shows up as nodular painless and asymptomatic slow-growing mass. Otherwise, ATs growing invasively into testicular parenchyma may show symptoms similar to other diseases like epididymitis and testicular sarcomas [3]. At the time of the excision the tumor has a dimension ranging from 2 to $5 \mathrm{~cm}$. Due to its indolent and silent nature, AT is diagnosed in patients from 18 to 72 years of age. Surgery excision is the election method to remove the tumor [4].

\section{Case Report}

A 59 years old patient presented with an omental painless palpable mass that was ultrasonogra- phically evaluated. The examination spotted a solitary firm nodule within a herniation sac. The gross examination of the excision biopsy showed greyish soft fragment of $3 \times 2 \times 2 \mathrm{~cm}$. HE stained sections of the nodule were histological analyzed. The neoplasm was made from epithelioid cell type arranged in dilated and irregular tubular structures lined by a flattened and cuboidal epithelium with an adenoid cystic pattern; neoplastic tubules were surrounded by a fibrous stroma [Fig.1]. Mucus material was filled epithelioid structures.

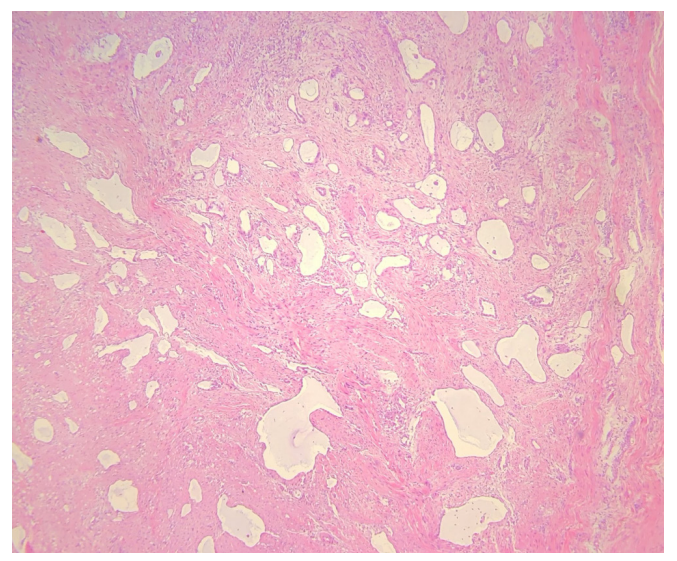

Fig.1: HE stained section of AT showing tubulo-cystic structures within a fibrous stroma. 
Immune-phenotype of the tubular structures (CD68-, PanK+, CD31-, calretinine $\mathrm{V}+, \mathrm{HBME1}+$ ) confirmed mesothelial origin of the neoplasm [Fig.2-4]. An additional marker (GRP78) was evaluated in the context of confront of benign mesothelial neoplasm with malignant mesothelioma [Fig.5].

\section{Discussion}

Only few cases of ATs arising outside genital tract have been reported including origin from pleura, liver, pancreas, omentum, adrenal glands. ATs usually grows as solitary fibrous nodules, indolent in nature. They are generally discovered as incidental findings during routine examination, surgery or autopsy. Although these types of tumors are benign, histologically they can present with a variety of morphologies that sometimes can represent a diagnostic challenge to distinguish

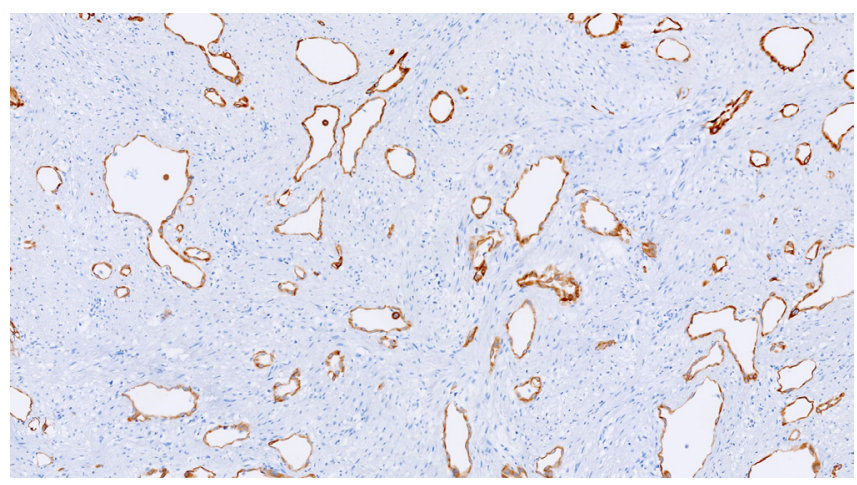

Fig.2: Mesothelial tubular structures CalretV+.

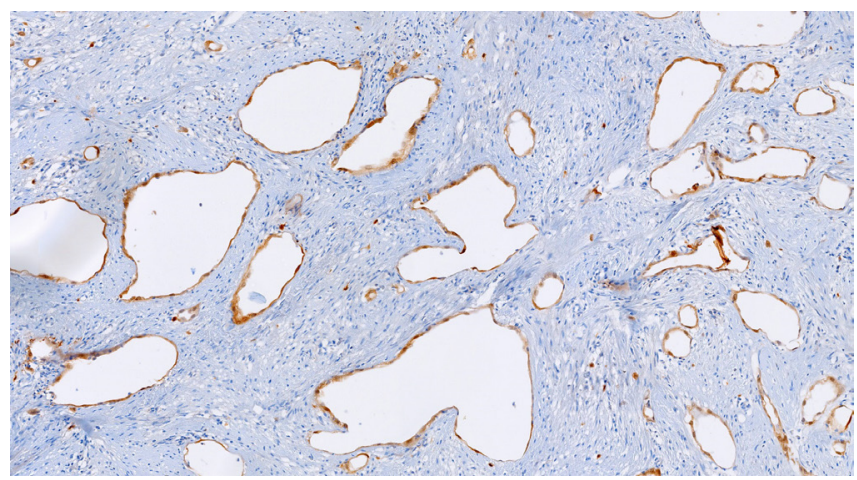

Fig.3: Mesothelial tubular structures PanK+. them from malignant entities. In general, omental origin of the nodule can increase the concern for a metastatic cancer. For example, omental ATs with signet ring cell feature can resemble a metastatic well differentiated adenocarcinoma, above all when cytologic atypia is detectable. However, circumscription of the neoplasm and absence of pleomorphism suggest the benign origin of the tumor. There is a debate about the cell type from which ATs arise: it has been proposed a mesothelial, mesonephric, müllerian and endothelial origin. Both IHC and ultrastructural findings suggest the mesothelial derivation of the tumor. Frequently, chronic inflammation and fibrosis accompany ATs and it has been established an association between insults to the peritoneum (like as hernial sac) and AT. These observations could hypothesize the hyperplastic nature of these lesion, but considering the mesothelial differentiation of the tumor, the

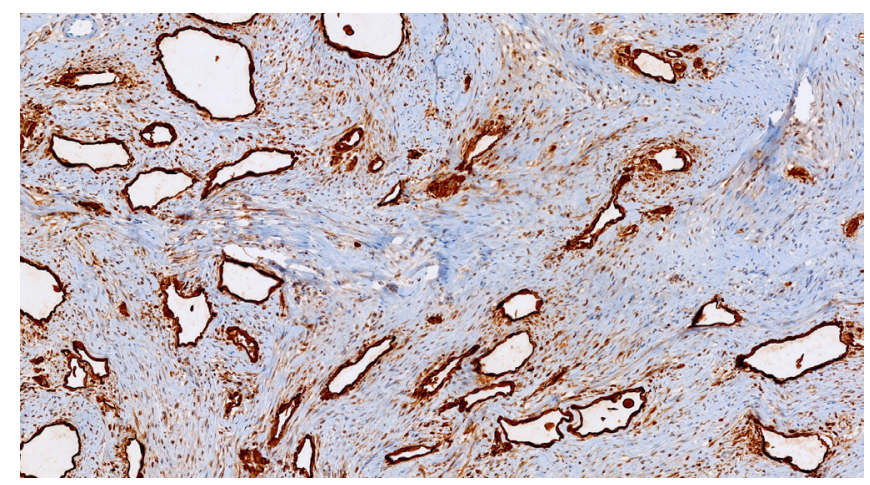

Fig.4: Mesothelial structures HBME-1+.

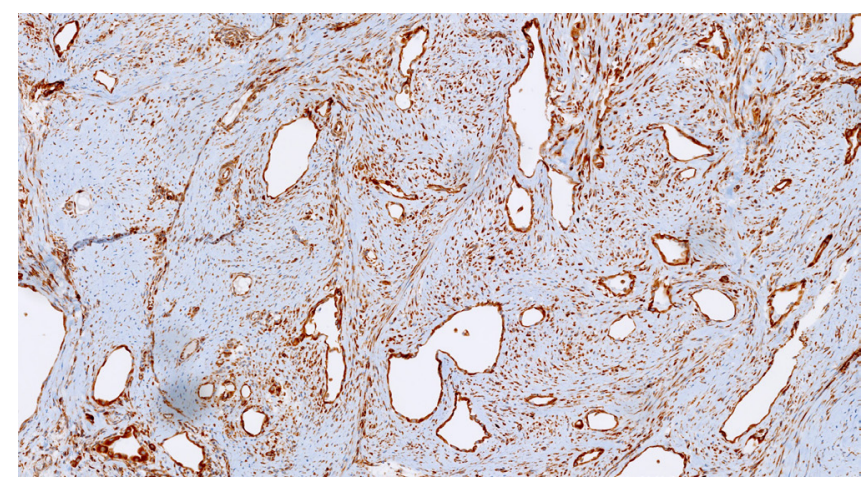

Fig.5: Mild expression of GRP78 in tubular and stromal component. 
absence of papillary architecture doesn't improve it [5].

GRP78 is an ubiquitary protein located in different cell compartments where it plays different roles. GRP78 is mainly known as a chaperon resident in the endoplasmic reticulum (ER); GRP78 can regulate the retro-translocation of malfolded proteins to the cytoplasm where they can be degraded by proteasome $26 \mathrm{~S}$. Another response mediated by this chaperone is the UPR, particularly involved in cancer survival. UPR can be inducted by accumulation of malfolded proteins in ER resulting in the ER stress. ER stress can be caused by oxidative stress, heat shock in normal cells. In tumoral cells activation of UPR can also depends on accumulation in the ER of polypeptides expressed by mutated alleles: these proteins cannot acquire the correct conformation determining the activation of GRP78. Once GRP78 triggers the UPR, it activates two kinase (PERK and IRE1) and promote the traslocation of the transmembrane protein ATF6 to the Golgi surface. A protease located on Golgi cleaves the cytosolic domain of ATF6, releasing a transcription factor. In summary PERK, IRE1 and ATF6 up-regulate UPR genes, that slow down the translation apparatus and redirect it to the expression of pro-survival genes. The same GRP78 translocates from ER to plasma membrane where it influences cell-survival via activation of the PI3K/Akt pathway. UPR shows how cancer can benefit from oxidative stress and genomic burden to increase his proliferation ability.

In an IHC perspective GRP78 is located mainly in the ER in normal cells: in ATs, like in benign mesothelial cells, GRP78 Ab stained only the cytoplasm and sustained this theory. In malignant mesothelioma (confronted with ATs), GRP78 Ab stained preferentially on cell surface, coherently with an un-mitigate activation of the PI3K/Akt pathway.

\section{Conclusion}

Extra-genital ATs are rare, needing a special attention when they interest peritoneal cavity to rule out secondary tumors. Microscopic morphology and tumoral immune-phenotype help to distinguish ATs from well-differentiated adenocarcinomas, especially in the context of a signet-ring cell type. This case represents also an occasion to confront the differences of GRP78 pattern expression between a benign and a malignant entity, both originating from the same cell type.

Contributors: SE: manuscript writing, patient management; $\mathrm{NE}$ : manuscript editing, patient management; AP: critical inputs into the manuscript. SE will act as a study guarantor. All authors approved the final version of this manuscript and are responsible for all aspects of this study.

Funding: None; Competing interests: None stated.

\section{References}

1. Williams SB, Han M, Jones R, Andrawis R. Adenomatoid tumor of the testis. Urology. 2004;63(4):779-781.

2. Overstreet K, Wixom C, Shabaik A, Bouvet M, Herndier B. Adenomatoid tumor of the pancreas: a case report with comparison of histology and aspiration cytology. Mod Pathol. 2003;16(6):613-617.

3. Chen D, Yu Z, Ni L, Gui Y, Yang S, Shi B, et al. Adenomatoid tumors of the testis: A report of two cases and review of the literature. Oncology Letters. 2014;7:1718-1720.

4. Amin W, Parwani AV. Adenomatoid tumor of testis. Clin Med Pathol. 2009;2:17-22.

5. Hayes SJ, Clark P, Mathias R, Formela L, Vickers J, Armstrong GR. Multiple adenomatoid tumours of the liver and peritoneum. J Clin Pathol. 2007;60:722-725. 